on the position and design of controls as well as on the accuracy required. An interesting table sets out the characteristics of various kinds of controls such as cranks, wheels, levers and push buttons, and examines their suitability for different tasks and loads. There is also a short section on power-assisted controls and the layout of machines.

\section{Phase-locked Lasers}

L. R. Enloe and J. K. Rodda of the Bell Telephone Laboratories (Bell Telephone Record, 42, 70, Feb. 1965) have succeeded in locking two lasers together in phase by means of a feedback loop which holds the phase difference between the waves generated by two singlefrequency $\mathrm{He}-\mathrm{Ne}$ lasers to within a third of a degree. The frequency of the lasers was approximately $5 \times 10^{14}$ $\mathrm{c} / \mathrm{s}$. The laser beams were combined by means of a halfsilvered mirror, which sent part of the combined beam to a screen and the remainder to a photomultiplier. The output current from the photomultiplier, being proportional to the instantaneous power of the combined light waves, increases when the light waves interfere constructively and decreases when they interfere destructively. Thus, the value of the instantanoous current depends on the phase difference between the two laser beams. The voltage produced by the current is fed back through a d.c. amplifier and a low pass filter to a piezoelectric transducer supporting one of the mirrors of the resonator of the receiving laser. The resonating length and correspondingly the frequency of the lasor is thus altered and forces the receiving laser to track the frequency and phase of the transmitting laser. Phase-lock was maintained even when the frequency of the transmitting laser was changed by $\pm 50 \mathrm{Mc} / \mathrm{s}$ and when, in addition, its beam was attenuated by $50 \mathrm{~dB}$. Phase-locking at optical frequencies permits the use of homodyne detection, with corresponding reduction in transmitted power, in experimental light communications systems, and also facilitates the investigation of interactions of light waves from separate sources.

\section{Journal of Experimental Social Psychology}

ACADEmic Press has recently published two new journals in psychology: one is the Journal of Experimental Research in Personality, the other the Journal of Experimental Social Psychology (1, No. I (January 1965). Edited by John Thibaut. Pp. 1-102. Subscription rates (4 issues): Institutional subseribers 16 dollars; Private subscribers certifying that the journal is for personal use 8 dollars. New York and London: Academic Press, 1965). These new journals have started at the same time as the well-known Journal of Abnormal and Social Psychology has split into two separate parts, one dealing with personality and social psychology, the other with abnormal psychology-just more than two years after the appearance of the British Journal of Social and Clinical Psychology. There is obviously a healthy ferment in this general area of the application of scientific method and experimental design to social problems, and the increasing sophistication and quality of the experiments reported make it appoar that the next few years will see a rapid advance in these areas. The composition of the editorial board suggests that the Journal of Experimental Social Psychology will deal largely with work on small groups, and the contents of the first issue do not disappoint this expectation. There is, for example, a paper on risk-taking in groups, which investigates the well-known phenomenon that decision-making in groups leads to enhanced risktaking as compared with individual decision-making. (The authors conclude their analytical investigation by saying that "The occurrence of group discussion is both necessary and sufficient for generating the risky shift effect.".) It is a pity that the authors have followed the usual habit American experimental psychologists of leaving out of any reference to personality and individual differences; as the work of Rim has shown, extroverts and introverts react quite differently in situations of this kind and the analysis of experimental investigations of small groups is incomplete when an important source of variance is neglected. Nevertheless, this paper like the others is of a high standard and the new Journal makes a welcome addition to the small group of standard-bearers favouring the experimental approach to social problems.

\section{Biological Luminous Clocks}

A RECENT general account, published in the Rockefeller Institute Review ( 3 , No. 1,$10 ; 1965)$, of the past few years work on the luminescent dinoflagellate Gonyaulax polyedra reveals some interesting details of the mechanism of bioluminescence and the nature of biological clocks. This marine plant is smaller and easier to cultivate than its better-known relative Noctiluca, and flashes of light are easily produced by shaking the cultures or bubbling air through them. It has been known for a long time that the amount of light produced waxes and wanes in synchrony with the alternation of day and night, and exact measurements show that the light is some 50-100 times stronger in the middle of the night than in the daytime. The rhythmicity persists when cultures are kept in continuous dim light (100 lux) but disappears under continuous bright light. Dr. J. W. Hastings and his colleagues in the United States find that the rhythm can be altered to a small extent by changing the periods of illumination, for example, to a 14-h 'day' of 7-h light and 7-h darkness, but that with shorter periods the plant reverts to the inherent, approximately $24 \mathrm{~h}$, rhythm. This rhythm can also be upset under continuous dim light by relatively short exposures $(2.5 \mathrm{~h})$ to brighter light, the cycle being either accelerated or retarded according to the stage at which the light stimulus is given. In addition to the luminescent rhythm, similar entrained $24-\mathrm{h}$ rhythms have been found in the processes of cell-division and photosynthesis, though with peaks at different times in the day. The question arises, therefore, as to whether the plants possess three different internal physiological 'clocks', or if there is one master process that is able to control the three separate rhythms. The same investigators have isolated active particles $(c .0 \cdot 5 \mu)$ from the cells, and find that flashes are emitted by lowering the $p H$. Although the real function of the luminescent reaction is still obscure, the authors believe that it is important to the organisms because of the high-energy intermediate products formed, and not because of the light emission.

\section{Beit Memorial Fellowships for Medical Research}

The following elections to Beit Memorial fellowships for medical research have been announced: Junior Fellowships, W. N. Adams Smith, to study the influence of sex hormones on the maturation of the brain, at the Department of Human Anatomy, University of Oxford; N. P. Bishun, to study autosomal and sex chromosome abnormalities associated with congenital anomalies in children and foetuses, at the Department of Anatomy, Queen's University, Belfast; M. R. Holloway, to study the mechanism of enzymatic oxidation and reduction of pyridine nucleotide coenzymes, at the Department of Biochemistry, University College, London; J. A. Kirrane, to study immunological interrelationships between collagens, at the Medical Research Council Rheumatism Research Unit, Taplow; R. Kumar, to study factors determining responses to psychomotor drugs, at the Department of Pharmacology, University College, London; Clare Bernadette McLoughlin, to study haemoglobin synthesis in thalassaemia and related disorders, at the Department of Chemical Pathology, University College Hospital Medical School, London; A. K. Singh, to study the absorption, transport and haemopoietic role of folic acid, at the 